\title{
Sensing Sound Pressure in an Anechoic Chamber Using Backscattered Laser Light
}

\author{
B. PIPER* AND T. KOUKOUlas \\ Acoustics Group, National Physical Laboratory, \\ Hampton Road, Teddington, TW11 0LW, Middlesex, United Kingdom
}

\begin{abstract}
Current standards for the measurement of the SI derived unit of sound-in-air pressure, the pascal, are based upon microphone reciprocity calibration and are achieved indirectly through microphone sensitivity. These methods require microphones of specific geometry and performance characteristics, effectively artefacts, and are traceable through standards for electrical and dimensional units. Measurement of acousto-optic interactions can provide a direct approach to measuring sound pressure. One acousto-optic interaction is the periodic scattering of photons caused by particles moving in a sinusoidal manner due to propagating sound across interference fringes formed at the intersection of two coherent laser beams. The sequence of these scattered photons, which is collected using telescopic optics and generated by a single photon counting device, can be autocorrelated to yield the periodicity of the photon events. Through mathematical analysis of the autocorrelation function it has been shown that acoustic particle velocity is inversely proportional to the time of the first minimum. This has effectively been shown for measurements in acoustic standing wave tubes and has been developed into a method which can be applied in an anechoic chamber. This paper describes the design and implementation of such a system which allows for a comparison of sound pressure measurements using optical and microphone based techniques.
\end{abstract}

DOI: 10.12693/APhysPolA.127.128

PACS: 06.20.-f, 42.62.Eh, 43.58.+z, 78.20.hb

\section{Introduction}

The current standards for microphone calibration are based on the reciprocity principle $[1,2]$. These standards require microphones of specific geometry and accurate modelling of transfer functions between acoustically coupled microphones. This is true for all types of calibration including free field. Since the standards are achieved through the use of reciprocal microphones pressure is indirectly realised with reference to electrical and dimensional standards. These special microphones can be considered calibration artefacts. They are analogous to the platinum-iridium blocks that form the current standard for the unit of mass, the kilogram. Within the field of metrology there is a strong initiative of defining all SI units in terms of fundamental constants. For the example of mass this could be a definition based on Planck's constant or Avogadro's number [3]. For the unit of sound this can be achieved through the use of optical techniques, in particular photon correlation spectroscopy (PCS), which would be traceable through the definition of the metre and the second.

\section{Photon correlation spectroscopy for measuring acoustic particle velocity}

There are a number of optical methods that exist for measuring flows in fluids. An acoustic field can be considered an oscillating flow and therefore some of these

${ }^{*}$ corresponding author; e-mail: ben.piper@npl.co.uk methods are applicable for measuring acoustic particle velocity. In the simplified environment of standing wave tubes laser Doppler anemometry (LDA) [4, 5] and velocimetry (LDV) [6], particle image velocimetry (PIV) [5] and PCS $[7,8]$ have all been applied to measure acoustic particle velocity with varying degrees of success. It has been shown that PCS can be applied with minimal seeding [9] meaning that the acoustic properties of the measurement medium are not changed and that it is possible to adapt this technique for use in a free field (anechoic) chamber [10, 11].

PCS is a technique which can be adapted to measure the periodicity of a sequence of photons that are scattered by two intersecting beams of coherent laser light. The intersection of two coherent beams creates optical fringes. As a particle moves through these fringes photons are scattered with a periodicity that is a function of the velocity of the particle and the spacing of the fringes. The time to the first minima, $\tau_{\min }$, of the autocorrelation function (ACF) of the photon sequence is directly related to the acoustic particle velocity, $u_{m}$, as shown in Eq. (1) [12], where $f$ is the acoustic frequency, $\lambda$ is the wavelength of the laser light and $\theta$ is the half angle of the intersection between two coherent lasers beams.

$$
u_{m}=\frac{3.832 f \lambda}{4 \sin (\theta) \sin \left(\pi f \tau_{\min }\right)} .
$$

Acoustic pressure can be calculated from the acoustic particle velocity using Eq. (2) where $\rho$ is the density of air and $c$ is the speed of sound in air, assuming the measurement point is in the acoustic far field

$$
p=\rho c u \text {. }
$$




\section{Photon correlation spectroscopy setup for measurements in a free-field chamber}

An overview of the PCS setup for measurements in a free-field chamber is shown in Fig. 1. The system can be separated into 4 main sections - the optical delivery system, the acoustic source and environment, the photon collection system, and the processing and control system. The components for the optical delivery and photon collection are both fixed onto a shared optical table which is placed outside the free field chamber. Inside the chamber the acoustic source is placed in one corner and aligned to the intersection of the two coherent laser beams. The acoustic velocity detected by a PCS system is the same for all directions of propagation as long as the measurement point is in the far field of the acoustic source. The setup is controlled from two signal generators and a PC fitted with a hardware correlation board.

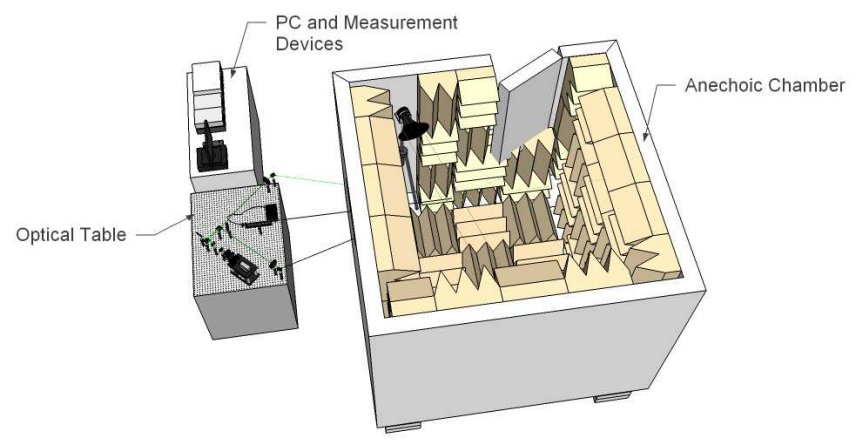

Fig. 1. Overview of PCS setup for measurements in a free-field chamber.

\subsection{Optical beam delivery system}

A significant challenge of applying PCS in a free chamber when compared to a standing wave tube is the need to focus and cross the laser beams several metres from the laser source whilst maintaining the quality and coherence of the beams. The system shown in Fig. 2 has been carefully designed to give a Gaussian interference region that has dimensions of $1 \mathrm{~mm} \times 3.4 \mathrm{~mm} \times 1 \mathrm{~mm}$ and a fringe spacing of $895 \mathrm{~nm}$. The laser unit is a frequencydoubled Nd:YAG source. The beam passes through a pair of plano-convex lenses, the first with a focal length of $22 \mathrm{~mm}$ and the second with a focal length of $80 \mathrm{~mm}$, which expand and then focus the beam at a distance of approximately $2 \mathrm{~m}$. The exact focal point is adjusted by changing the distance between the two lenses. The beam is then routed through a polarising beam splitter that, in conjunction with a wave plate placed in front of the laser source, removes any trace of horizontally polarised light. This is followed by a second beam splitter which gives two beams that are separated by $90^{\circ}$. These are directed to the intersection point inside the chamber using a pair of mirrors, through small openings in the chamber walls. The half angle of intersection is $17.3^{\circ}$ and the optical intensity of the volume is approximately $200 \mathrm{~mW}$.

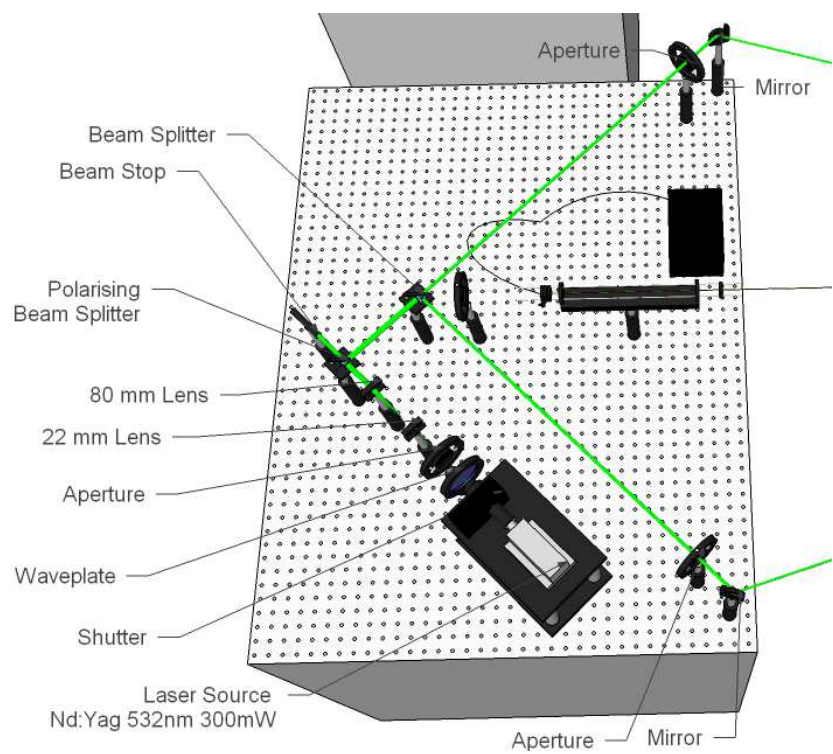

Fig. 2. Optical beam delivery system.

\subsection{Acoustic source}

The minimum acoustic pressure that can be detected using this system is defined by the measurement window and increases with frequency. For the audible range of frequencies the minimum detectable sound pressure level (SPL) ranges from $98 \mathrm{~dB}$ at $500 \mathrm{~Hz}$ to $123 \mathrm{~dB}$ at $20 \mathrm{kHz}$. In order to generate these pressures at the intersection point whilst maintaining spherical radiation a small and highly efficient source is needed. A Faital Pro HF14AT compression driver coupled to a Faital Pro LTH142 horn is used which has a sensitivity ( $1 \mathrm{~W}$ at $1 \mathrm{~m})$ of $106^{-}$ $110 \mathrm{~dB}$ between $500 \mathrm{~Hz}$ and $20 \mathrm{kHz}$. The speaker is driven by an audio power amplifier which is connected to a function generator.

\subsection{Photon collection system}

Figure 3 shows the layout of the photon collection system. A Keplerian telescope attached to a tilting platform is focussed on the interference region inside the chamber through an opening in the wall. The image collected by this telescope is focussed into a single mode optical fibre using an aspheric lens. The fibre couples the collected

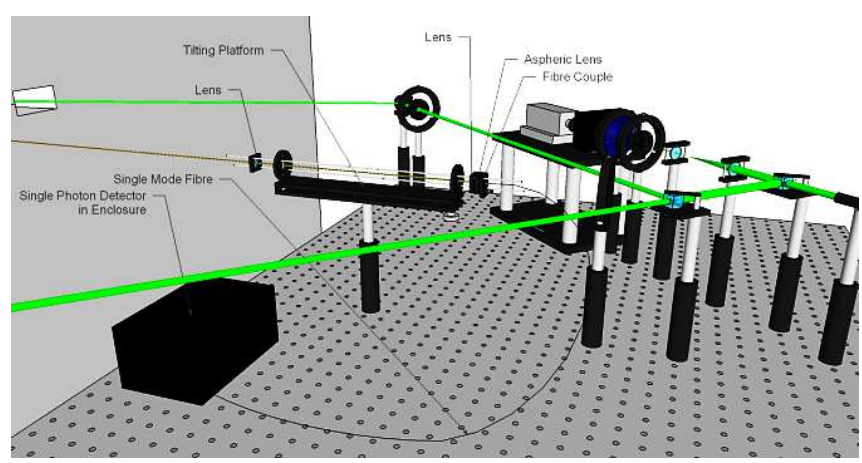

Fig. 3. Photon collection system. 
photon sequence to a photon counting module via a collimator. The output of this module is a sequence of TTL pulses, each pulse corresponding to a single photon event, which are measured using a correlation board. The results are then analysed in software as will be discussed.

\subsection{Mean flow and gating}

In a standing wave tube the acoustic particle velocity at a point is fixed and there is very little mean flow. It is appropriate to measure a continuous ACF as the measurement is focussed on a single velocity component. In free field conditions the acoustic velocity at a single point is sinusoidal in nature and therefore a continuous ACF would give the mean velocity of the acoustic signal, assuming not any acoustic velocity components were present. Due to the volume of air in a free field the ambient mean flow is significant in magnitude and will affect the measurement of acoustic particle velocity. The velocity of the mean flow is superimposed on the acoustic velocity such that the velocity in one half of a sine wave is increased and the other half reduced. Because the ACF can only give the magnitude of the velocity and not the direction the mean flow velocity and the acoustic velocity cannot be separated for a continuous measurement. In order to decouple the acoustic velocity from the mean flow the correlator board is controlled using a gating signal. By measuring an ACF for $\frac{1}{4}$ of the acoustic cycle centred on the positive and negative peaks the mean flow can be cancelled out as its contribution is the difference between the 2 ACFs. Averaging alternate measurements of the positive and negative velocity peaks allows the mean flow to be decoupled on a short time scale. As this limits the measurement window to $\frac{1}{4}$ of the acoustic wavelength the acoustic pressure required to measure an $\mathrm{ACF}$ increases with acoustic frequency.

\subsection{Example of a measured $A C F$}

Figure 4 shows an example of an ACF measured for an acoustic signal of $1 \mathrm{kHz}$. The time to the first minima is $18.66 \mu \mathrm{s}$ which corresponds to an SPL of $112.6 \mathrm{~dB}$.

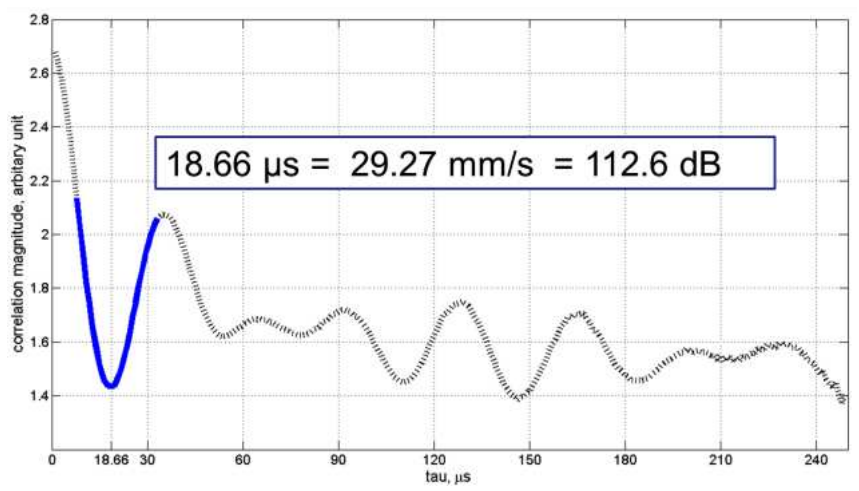

Fig. 4. Measured autocorrelation function for a $1 \mathrm{kHz}$ sinusoid with first minimum used for fitting highlighted in bold.
In order to accurately identify the time to the first minimum a spline interpolation fitting function is used and the section to which this is applied is highlighted in Fig. 4.

\section{Comparison of microphone sensitivity measured using PCS and pressure reciprocity}

In order to assess the performance of the measurement system the sensitivity of a Bruel and Kjaer 4134 microphone was measured and compared to the sensitivity of the microphone measured using pressure reciprocity. For each frequency 6 ACFs were measured and the results averaged. The random error of the measurements was less than $1 \%$ except for at $15850 \mathrm{~Hz}$ where it was $1.6 \%$. The microphone was then placed such that the centre of its diaphragm was at the laser beam intersection point and aimed at the centre of the acoustic source. The output of the microphone was measured using an oscilloscope and then the sensitivity was calculated based on the pressure measured using the optical system.

TABLE

Comparison of LS2 microphone sensitivity measured using pressure reciprocity and photon correlation spectroscopy at $1 / 3$ octave band centre frequencies.

\begin{tabular}{r|c|c|c}
\hline \hline \multirow{1}{*}[\mathrm{Hz}]{} & $\begin{array}{c}\text { Sensitivity } \\
\text { measured } \\
\text { with pressure } \\
\text { reciprocity } \\
\text { [dB }\end{array}$ & $\begin{array}{c}\text { Sensitivity } \\
\text { measured } \\
\text { using PCS }\end{array}$ & $\begin{array}{c}\text { Difference } \\
{[\mathrm{dB}]}\end{array}$ \\
\hline 501 & -39.89 & -40.57 & 0.68 \\
631 & -39.89 & -40.25 & 0.37 \\
794 & -39.87 & -39.91 & 0.04 \\
1000 & -39.84 & -40.17 & 0.33 \\
1259 & -39.80 & -39.94 & 0.15 \\
1585 & -39.73 & -39.98 & 0.25 \\
1995 & -39.62 & -40.05 & 0.43 \\
2510 & -39.44 & -39.24 & -0.21 \\
3160 & -39.18 & -39.58 & 0.40 \\
3980 & -38.77 & -38.81 & 0.04 \\
5010 & -38.14 & -37.59 & -0.55 \\
6310 & -37.22 & -36.68 & -0.54 \\
7940 & -35.90 & -35.23 & -0.67 \\
10000 & -34.16 & -33.48 & -0.68 \\
12590 & -32.24 & -30.97 & -1.27 \\
15850 & -30.91 & -29.85 & -1.06 \\
& & &
\end{tabular}

Table shows a comparison between the sensitivities measured with each method. As the optical measurements were made in a free field and in order to compare like with like, the standard free field correction [13] was applied to sensitivity measured using the pressure reciprocity technique. The agreement between the sensitivities is within $1 \mathrm{~dB}$ up to $10 \mathrm{kHz}$. There is a trend at the higher frequencies for PCS to measure an increasingly higher sensitivity than pressure reciprocity. This could be due to a number of factors including uncertainty 
in the free field correction applied to the microphone measurements, differences in the harmonic distortion measured by the microphone and the optical system, misalignment of the microphone, particle inertia, artefacts of the chamber and microphone mounting and non-linearity of the transducers. Further investigation is needed in order to understand these differences, which is the next key step in establishing new primary standards based on the optical method described here.

\section{Conclusion}

This paper described an optical system based on PCS that can be used to measure the sound pressure at a point inside a free-field chamber with full description of optical, acoustic, and signal processing given. The advantage of such a measurement system is the ability to measure sound pressure in an absolute and non-invasive way with traceability to the definition of the metre. A comparison between a microphone sensitivity measured using PCS and pressure reciprocity is given where the agreement between the two methods is good. There is a trend at the higher frequencies for the optical method to measure a higher sensitivity and this could be due to a number of factors which need further investigation.

\section{Acknowledgments}

(C)Crown Copyright

Queen's Printer and Controller of HMSO, 2014.

\section{References}

[1] Standard IEC 61094-2, 2009.

[2] Standard IEC 61094-3, 1995.

[3] T.P. Hill, J. Miller, A.C. Censullo, Metrologia 48, 83 (2011).

[4] M.W. Thompson, A.A. Atchley, J. Acoust. Soc. Am. 117, 1828 (2005).

[5] M. Campbell, J.A. Cosgrove, C.A. Greated, S. Jack, D. Rockliff, Opt. Laser Technol. 32, 629 (2000).

[6] A. Degroot, R. MacDonald, O. Richoux, B. Gazengal, M. Campbell, Appl. Acoust. 69, 1308 (2008).

[7] D. Hann, C.A. Greated, Meas. Sci. Technol. 5, 157 (1994).

[8] T. MacGillivray, D. Campbell, C. Greated, R. Barham, Acta Acust. 89, 39 (2003).

[9] T. Koukoulas, P.D. Theobald, T. Schlicke, R.G. Barham, Opt. Lasers Eng. 46, 791 (2008).

[10] T. Koukoulas, B. Piper, P.D. Theobald, J. Acoust. Soc. Am. 133, EL156 (2013).

[11] B. Piper, T. Koukoulas, A. Torras-Rosell, P. Theobald, Proc. Inst. Acoust. 35, 207 (2013).

[12] J.P. Sharpe, C.A. Greated, J. Phys. D Appl. Phys. 22, 1429 (1989).

[13] Standard IEC 61094-7, 2006. 